\title{
Besouros, parasitóides e Ipomoea \\ (Convolvulaceae): Um estudo sobre discriminação de hospedeiro
}

\author{
C. Ronald Carrol (")
}

\begin{abstract}
Resumo
O besouro Stolas sp. (família Chrysomelidae), herbívoro tanto quando larva como adulto, preda a trepadeira Ipomoea asarifolia. Ao redor de $\mathrm{Ma}$ naus, em média $86 \%$ de seus agrupamentos de ovos foram completamente parasitados pela vespa Emersonella neveipes (Eulophideae). A vespa é forética em besouros fêmeas. Em comparação com besouros do campo que não possuem vespas foréticas, os besouros em associação com vespas apresentam as seguintes características: (1) produziram ovos mais cedo, (2) produziram mais agrupamentos de ovos e (3) tiveram esperança de vida, em laboratório, mais curta. E. neveipes também ataca Chelymorphe cassidea. Como Stolas $\mathrm{sp}$. nẫo se alimenta de folhagem de batata-doce e não ocorre em campo onde há batata-doce, poderia ser um hospedeiro substituto, inofensivo e útil, para o Emersonella neveipes. Como algumas espécies de Convolvulaceae são importantes ervas daninhas para as plantações, os besouros Cassidenae, que comem a sua folhagem, podem ter um papel útil na supressão dessas ervas daninhas. Qualquer programa de con. trole biológico, para a supressão das pestes que atacam a batata-doce, deve favorecer a prohferaçāo das ervas daninhas nocivas que sejam controladas pela fauna herbivora das batatas-doces.
\end{abstract}

\section{INTRODUÇÃo}

O autor está atualmente pesquisando o papel desempenhado pela vegetação natural, tanto como uma fonte herbivora quanto como uma fonte e refúgio para predadores e parasitas úteis, na ecologia das plantações tropicais de pequena escala e, principalmente, as culturas tropicais de subsistência. Este trabalho relata uma interação hospedeiro-parasita na Bacia Amazônica do Brasil, a qual envolve a trepadeira nativa neotropical, Ipomoea batatas.

A trepadeira tropical, Ipomoea asarifolia (Roem et Schult) [Convolvulaceae] é uma espécie pioneira de áreas altamente mexidas pelo homem, como as margens de rodovias, margens de rios e vegetação marginal de terras onde se pratica agricultura. Ipomoea asarifolia parece ser uma especialista em colonização rápida, de terrenos que foram roçados, e desaparece rapidamente quando esses terrenos são invadidos por espécies de plantas mais competitivas. A trepadeira geralmente desaparece da sucessão vegetal muito antes que surjam espécies lenhosas. Ipomoea asarifolia ocorre freqüentemente, ao longo aas margens de campos de agricultura, em íntima proximidade com seu parente, importante para a agricultura, a batata doce.

Os herbívoros mais comuns da Ipomoea asarifolia são as várias espécies de besouros Cassidíneo, um besouro Chrysomelideo e várias lagartas de lepidópteros. Os besouros Cassidinae da Ipomoea asarifolia, pelo menos na vizinhança de Manaus, aiimentam-se somente dessa hospedeira e, às vezes, também, de outras espécies de Convolvulaceae como, por exemplo, Merremia umbellata. As Convolvulaceae são bem representadas na região amazônica. Falcão (1971) relata 19 gêneros e pelo menos 40 espécies nessa região. Dez dessas espécies pertencem ao gênero Ipomoea. Nosso conhecimento sobre a fauna herbívora dessas espécies é escasso. Não obstante ser a batata doce importante para a agricultura da região, Silva (1967) enumera, para todo o Brasil, 12 gêneros e 24 espécies de besouros Cassidieos que se alimentam de Ipomoea, embora todos, excếo três espécies, sejam pestes da batata doce. Os besouros Cassidinae não estão limitados, em sua alimentação, às Convolvulaceae. embora as espécies herbáceas dessa familia de plantas formem o maior número de hospedeiros para os besouros Cassidinae. Quando Blackwelder (1957) complicou sua exaustiva lista de besouros neotropicais, haviam

(") - Department of Ecology and Evolution, SUNY - Stony Brook, U.S.A. Pesquisador visitante do INPA, 1976. 
1570 espécies conhecidas de Cassidinae na América do Sul. Isto deve representar apenas uma fração do número real de espécies, já que cinco das nove espécies de besouros Cassidinae coletados, da Ipomoea asarifolia, neste estudo, parecem ser espécies não descritas.

Este trabalho visa principalmente uma espécie Cassidinae não descrita (Stolas $\mathrm{sp}$ ), que é o inseto que mais comumente se alimenta das folhas de Ipomoea asarifolia e com as vespas parasitas (Emersonella neveipes) que atacam os ovos desse e de outros besouros.

\section{DESCRIÇÃO DOS LUGARES DE ESTUdO}

$\mathrm{O}$ estudo foi desenvolvido dentro de $50 \mathrm{~km}$ de Manaus. Oito sítios foram usados. Estes lugares são denominados: $\mathrm{Km} 26$ (1), $\mathrm{Km} 26$ (2), Km 26 (3), Km 42,7, Campo, V-8, Caminho do Poço e Portão. A quilometragem marcada nos sítios Km 26 (1), Km 26 (2), Km 26 (3) e $\mathrm{Km} \mathrm{42,7}$ refere-se às distâncias de Manaus ao longo da BR 010 . O Km 26 marca a entrada da reserva florestal Ducke, que é uma das reservas do INPA. O sítio V-8 refere-se ao nome de uma avenida que está ligada à BR 010, dentro da cidade de Manaus. Esses 5 (cinco) sítios têm vegetação baixa misturada com discretos trechos de I. asarifolia. Essa vegetação ao lado das estradas, margeia florestas secundárias em vários estágios de degradação.

O sítio "Campo" é um pasto coberto de ervas daninhas, com vários hectares de extenção, e forma fronteira com floresta secundária por dois lados e com um pequeno povoado e uma estrada pelos outros dois lados. O sítio está localizado a aproximadamente $100 \mathrm{~m}$ da estrada da sede do INPA e a aproximadamente $7 \mathrm{~km}$ do centro com portuário de Manaus. I. asarifolia encontra-se dispersa pelo campo e, normalmente, está intercalada com um grande número de outras espécies herbáceas, ao invés de ocorrer numa área determinada como nos outros sítios. O "Caminho do Poço" é um caminho sinuoso através de floresta secundária e de terra pantanosa que fica adjacente ao sítio "Campo". I. asarifolia ocorre aqui como uma linha contínua e estreita ao lado do caminho. O "Portão" é uma área de I. asarifolia de

aproximadamente 50 metros quadrados que fica adjacente ao portão do INPA.

Todos os sítios ocorrem sobre latossolos vermelhos e o clima da região é caracterizado por chuvas anuais $2.000 \mathrm{~mm}$.

Essas áreas de $I$. asarifolia são efêmeras. Estas espécie de Ipomoea invade a terra descoberta que resulta da completa derrubada da vegetação, mas é rapidamente eliminada quando outras espécies se estabelecem. Atualmente I. asarifolia ocorre mais comumente em sítios que estão sujeitos à freqüente interferência do homem, como ao longo de margens de estradas e nos limites de terras cultivadas pela agricultura. Mesmo durante o decorrer relativamente curto deste estudo, três dos sítios (Campo, Portão e Km 26 (1)) foram limpas de toda a vegetação.

\section{Resultados}

HERBÍVOROS

A percentagem média de área foliar da I. asarifolia que é removida pela Stola sp varia de sítio para sítio com um valor inferior ao redor de $5 \%$ e um valor superior ao redor de $25 \%$ (Tabela 1). Há uma correlação significativa entre o número de ordem da diversidade das espécies encontradas em cada sítio e os seus índices associados de dano foliar, sugerindo um aumento de pressão herbívora sobre a $I$. asarifolia com função do aumento da idade da comunidade. É claro que um aumento da atividade herbívora diminuiria a habilidade

TABELA 1 - Lista dos lugares no campo, em ordem crescente de diversidade de plantas (uma aproximação do estágio de sucessão), com o índice associado médio de dano foliar para cada sítio $\left(r_{s}=0,85\right.$ p $\left.<0,05\right)$.

Sítio

$\mathrm{V}-8$

$\mathrm{Km} 42,7$

$\mathrm{Km} 26$ (3)

$\mathrm{Km} 26$ (2)

Caminho do Poço

Campo

$\mathrm{Km} 26$ (1)
Indice de dano foliar
1,19

1,73

1,89

1,27

2,01

2,63

3,13 
TABELA 2 - Estrutura da população de Stolas sp e número total de indivíduos entre todos os outros besouros. Os besouros foram contados em amostras ao acaso de 2.000 folhas de cada sítio

\begin{tabular}{|c|c|c|c|c|c|c|c|c|c|c|}
\hline Sítio & $\begin{array}{l}\text { Fêmeas } \\
\text { adultas }\end{array}$ & $\begin{array}{l}\text { Machos } \\
\text { adultos }\end{array}$ & $\begin{array}{l}\text { Pares } \\
\text { em } \\
\text { cópula }\end{array}$ & $\begin{array}{l}\text { Ninhados } \\
\text { de ovos }\end{array}$ & $\begin{array}{l}\text { Total } \\
\text { de ovos }\end{array}$ & $\begin{array}{l}\text { Larvos } \\
1-3 \mathrm{~mm}\end{array}$ & $\begin{array}{l}\text { Larvos } \\
4-6 \mathrm{~mm}\end{array}$ & $\begin{array}{l}\text { Larvos } \\
7-10 \mathrm{~mm}\end{array}$ & $\begin{array}{l}\text { Pupos } \\
\text { vivos }\end{array}$ & $\begin{array}{c}\text { Total } \\
\text { de outros } \\
\text { besouros }\end{array}$ \\
\hline V-8 & 12 & 16 & 4 & 7 & 231 & 0 & 0 & 0 & 0 & 6 \\
\hline $\mathrm{Km} \quad 42,7$ & 10 & 3 & 1 & 8 & 243 & 202 & 107 & 0 & 2 & 2 \\
\hline $\mathrm{Km} \mathrm{26(3)}$ & 10 & 13 & 2 & 16 & 616 & 228 & 128 & 9 & 0 & 4 \\
\hline $\mathrm{Km} \mathrm{26(2)}$ & 4 & 1 & 1 & 2 & 51 & 6 & 5 & 6 & 0 & 2 \\
\hline Caminho do Poço & 17 & 25 & 4 & 16 & 504 & 91 & 5 & 3 & 0 & 2 \\
\hline Campo & 27 & 36 & 7 & 13 & 323 & 0 & 1 & 1 & 1 & 6 \\
\hline $\mathrm{Km} \mathrm{26(1)}$ & 9 & 2 & 0 & 10 & 304 & 42 & 30 & 0 & 1 & 3 \\
\hline
\end{tabular}

competitiva da I. asarifolia e aceleraria sua extinção local. Nắo há, entretanto, uma correlação significativa entre a média de dano foliar e o número de besouros em todos os estágios alimentares, que foram recenseados em cada sitio.

Stolas sp foi o herbivoro mais freqüentemente observado em todos os sítios. Exceto no sítio V-8, Stolas sp representou mais de $20 \%$ dos indivíduos de todos os besouros que estavam realmente comendo. Lepidópteros comedores de folhas, tal como a mariposa Agrius cingulata (Sphingidae), foram escassos em comparação com os besouros Chrysomelidae, e não foram encontrados durante nenhuma das coletas de folhas. Minadores de foIhas, embora freqüentes, foram excluídos deste estudo.

\section{PARASITISMO}

A maioria (média $86 \%$ ) dos ovos de Stolas sp foi parasitada pela vespa Emersonella neveipes (Elilophidade) (Tabela 3 ) e muitas pupas foram mortas (provavelmente por pentatomídeos) ou parasitadas pelas vespas Brachymeria sp e Spilachaleis sp (Chalcididae). Stolas sp desova em lotes de 10 a mais de 50 ovos com um número médio entre 30 e 40 . Lotes de ovos ou não apresentam parasitismo ou apresentam parasitismo completo. Daqueles ovos com parasitas, muitas das larvas jovens provavelmente são vítimas de formigas; em quatro ocasiões, larvas mais velhas foram comidas por espécies comuns de pentatomídeos.
As formigas são atraídas para as folhas por glândulas de néctar extraflorais. Predação e outras formas de mortalidade, provavelmente, contribuem para o padrão heterogêneo do número de larvas por foiha (Tabela 4) (do que tendências diferente de dispersão).

O enorme declínio em número das larvas grandes, por folha, poderia ser atribuído a combinações de 4 causas: (1) períodos menores de desenvolvimento para estágios (instais) larvais maiores; (2) dispersão de larvas maiores; (3) superposição incompleta de geraçōes e (4) mortalidade. Classes de tamanho de larvas foram escolhidas para refletir tempos semelhantes de desenvolvimento no laboratório, por isso os periodos relativos de desenvoivimento para as três classes larvais provavelmente não são muito diferentes no campo.

TABELA 3 - Porcentagem de ninhadas de ovos coletados no campo, que produziram parasitóides. NA significa que o tamanho da amostra foi menor que dez.

\begin{tabular}{lcl}
\hline \multicolumn{1}{c}{ Sítio } & $\begin{array}{l}\text { Número de } \\
\text { Ninhadas de ovos }\end{array}$ & \% Parasitados \\
\hline V-8 & 13 & 85 \\
Km 42,7 & 23 & 74 \\
Km 26(3) & 16 & 88 \\
Km 26(2) & NA & NA \\
Caminho do Poço & NA & NA \\
Campo & 55 & 85 \\
Km 26(1) & NA & NA \\
Portão & 0 & 100 \\
\hline
\end{tabular}


TABELA 4 - Número de larvas de Stolas sp por folha, como função de três classes (pequeno $=1-3 \mathrm{~mm}$, médio $=4-6 \mathrm{~mm}$, grande $=7-10 \mathrm{~mm}$ ). A Média e o Coeficiente de Variação foram calculados dos dados brutos (originais). O tamanho da amostra é de 2.000 folhas escolhidas ao acaso em cada sítio.

\begin{tabular}{|c|c|c|c|c|c|c|}
\hline \multirow{2}{*}{$\begin{array}{c}\text { Sítio } \\
\mathrm{Km} \mathrm{26(3)}\end{array}$} & \multicolumn{2}{|c|}{ Pequeno } & \multicolumn{2}{|c|}{ Médio } & \multicolumn{2}{|c|}{ Grande } \\
\hline & F & $\mathrm{N}$ & $\mathrm{F}$ & $\mathrm{N}$ & $\mathrm{F}$ & $\mathrm{N}$ \\
\hline & 12 & $1-5$ & 18 & $1-5$ & 8 & $1-5$ \\
\hline & 1 & $6-10$ & 3 & $6-10$ & 0 & $6-10$ \\
\hline & 4 & $11-15$ & 0 & $11-15$ & 0 & $11-15$ \\
\hline & 5 & $>15$ & 3 & $>15$ & 0 & $>15$ \\
\hline & $\underset{\mathrm{CV}}{\bar{X}}$ & $\begin{array}{r}9.91 \\
106.66\end{array}$ & $\begin{aligned} \bar{x} & = \\
\mathrm{CV} & =\end{aligned}$ & $\begin{array}{r}5.33 \\
168.67\end{array}$ & $\begin{array}{l}\bar{x}= \\
\mathrm{cV}=\end{array}$ & $\begin{array}{r}1.13 \\
30.97\end{array}$ \\
\hline
\end{tabular}

\begin{tabular}{|c|c|c|c|c|c|c|}
\hline \multirow[t]{6}{*}{$\mathrm{Km} 42.7$} & $\mathrm{~F}$ & $\mathbf{N}$ & $\mathrm{F}$ & $\mathrm{N}$ & $\mathrm{F}$ & $\mathbf{N}$ \\
\hline & 11 & $1-5$ & 18 & $1-5$ & 0 & $1-5$ \\
\hline & 2 & $6-10$ & 5 & $6-10$ & 0 & $6-10$ \\
\hline & 2 & $11-15$ & 2 & $11-15$ & 0 & $11-15$ \\
\hline & 5 & $>15$ & 1 & $>15$ & 0 & $>15$ \\
\hline & $\underset{\mathrm{CV}}{\bar{x}}$ & $\begin{array}{r}10.10 \\
111.29\end{array}$ & $\underset{C V}{\bar{x}}$ & $\begin{array}{r}4.12 \\
114.81\end{array}$ & $\begin{array}{c}\bar{x} \\
\mathrm{CV}\end{array}$ & $\frac{-}{-}$ \\
\hline
\end{tabular}

\begin{tabular}{|c|c|c|c|c|c|c|}
\hline \multirow{7}{*}{$\begin{array}{l}\text { Caminho } \\
\text { do Poço }\end{array}$} & $F$ & $\mathrm{~N}$ & F & $\mathrm{N}$ & $\mathrm{F}$ & $N$ \\
\hline & 6 & $1-5$ & 4 & $1-5$ & 2 & $1-5$ \\
\hline & 2 & $6-10$ & 0 & $6-10$ & 0 & $11-15$ \\
\hline & 1 & $11-15$ & 0 & $11-15$ & 0 & $6-10$ \\
\hline & 2 & $>15$ & 0 & $>15$ & 0 & $>15$ \\
\hline & $\bar{x}=$ & 8.27 & $\bar{x}=$ & 1.25 & $\bar{x}=$ & 1.50 \\
\hline & $\mathrm{CV}=$ & 36.03 & $\mathrm{CV}=$ & 40.00 & $\widehat{C V}=$ & - \\
\hline
\end{tabular}

A planta comestível, I. asarifolia, existe durante o ano inteiro e o herbário do INPA contém amostras de espécies de várias épocas durante o ano, com um dano foliar que é indistinguível do dano característico causado pela Stolas sp. Isso sugere que o besouro é ativo durante o ano inteiro, embora isso demonstre que as populações, na época do recenseamento, estivessem constantemente se reproduzindo. Os altos índices de parasitismo de ovos e pupas (Tabela 3 e 5) e a abundância de reduviideos, pentatomídeos e formigas no local, sugerem que a mortalidade por predação e parasitismo desempenha o papel principal no declínio do número de ovos, larvas e pupas.

\section{DISCRIMINAÇÃO DE HOSPEDEIRO}

Os parasitóides de ovos foram freqüentemente observado no campo sentados sobre o élitro de seus hospedeiros, os besouros Cassidinae, Stolas sp e Botanochara pavonia Boh. As vespas foram observadas somente em besouros fêmeas e a alta freqüência de múltiplas vespas por besouro (Tabela 6) implica que certos besouros podem ser mais atraentes ou mais facilmente escolhidos do que outros. Em diversas ocasiões as vespas foram vistas desovando em massas de ovos mesmo antes que os besouros terminassem sua postura. Estas vespas não se limitavam a ovos recentemente depositados, pois, no laboratório, ovos de dois dias de idade são sujeitos a serem parasitados com sucesso. Todas as vespas foréticas que foram observadas $(N=21)$ eram fêmeas e, portanto, as vespas não estavam formando par nem se acasalando sobre os besouros. Em duas ocasiões 4 vespas foram observadas sobre um único besouro. A probabilidade de ocorrência de 4 vespas sobre um mesmo besouro, por acaso, assumindo distribuições binomial, é $24 \times 10^{-5}$. O coeficiente de dispersão para essa associação é 25 , onde o valor para 1 ou mais indica agrupamento preferencial. Portanto, as vespas estão distribuídas de acordo com um padrão em que apresentam alta preferência por certas besouras. Coletamos no campo besouras com vespas foréticas e as besouras sem vespas mais próximas; foram trazidas ao laboratório e alimentadas com folhas frescas de Ipomoea. As besouras que estavam associadas com vespas depositaram ovos mais cedo do que as besouras sem vespas e, além disso, quase metade

TABELA 5 - Os dados obtidos em laboratórios para as pupas de Stolas coletadas no sítio Campo.

Pupas de Stolas sp

\begin{tabular}{cccc}
\hline Besouros & Parasitóides & \multicolumn{2}{c}{ Mortos } \\
\hline 5 & 11 & 24 & $\mathrm{~N}=40$
\end{tabular}

NOTA: Năo estāo incluídas muitas ocasiōes nas quais os fragmentos de pupas foram encontrados; o destino dessas pupas nâo pôde ser determinado. 
das besouras que não estavam associadas com vespas não puseram ovos nem em 7 dias $(F i$ gura 1). Besouras associadas com vespas produziram significativamente mais ninhos de ovos do que as besouras que não estavam associadas com vespas (Tabela 7). Se considerarmos apenas os indivíduos que estảo em fase de pôr ovos, teremos que as besouras associadas com vespas produzem mais massas (ninhos) de ovos do que o produzido por besouras não associadas. O número médio de ovos por ninho entre os dois grupos de besouros é, estatisticamente, igual.

A sobrevivência das besouras no laboratório é mais alta entre aquelas besouras que não estavam associadas com vespas foréticas (Tabela 8).

\section{DISCUSSÃo}

DISCRIMINAÇÃO DE HOSPEDEIRO

A discriminação feita pelo parasitóide ds ovos, Emersonella neveipes, de hospedeiros adequados de Stolas sp, parece ter dois componentes: (1) seleção do sexo apropriado do hospedeiro e (2) seleção de besouras que estão próximas da desova. A proporção média de sexo dos adultos Stolas $\mathrm{sp}$ coletados no campo foi 22 machos para cada fêmea. Em nenhum caso uma vespa esteve associada com um besouro macho e a probabilidade de que tais associações ocorressem somente com be-
TABELA 6 - O número de foréticos, parasitóides de ovos (todas as fêmeas) por besouro fêmea. A freqüência esperada de 4 vespas sobre um besouro, assumindo encontros ao acaso é $2.4 \times 10^{-5}$.

Número de vespas foréticas por besouro fêmea

\begin{tabular}{lccccc} 
Número de & 0 & 1 & 2 & 3 & 4 \\
besouros & 125 & 4 & 3 & 1 & 2 \\
Coeficiente de dispersão & \multicolumn{2}{c}{$\mathrm{S} 2 / \overline{\bar{x}}=2.50$} & &
\end{tabular}

souros fêmeas por acaso é muito pequena. Portanto, o parasitóide de ovos provavelmente discrimina fortemente em favor de hospedeiros fêmeas .

O segundo componente é mais complice do. Sob condições de laboratório os besouros fêmeas coletados no campo que estavam associados com vespa têm as seguintes características quando comparadas com besouros fêmeas que não estavam associadas com vespas no campo: besouras associadas com vespas (1) produzem ovos mais cedo, (2) produzem mais ninhadas de ovos e (3) têm uma expectativa de vida mais curta em laboratório. A terceira característica sugere que as vespas estão associadas com besouras mais veihas.

A explicação mais simples, consistente com as características de laboratório acima citadas, é que as vespas simplesmente se tor-
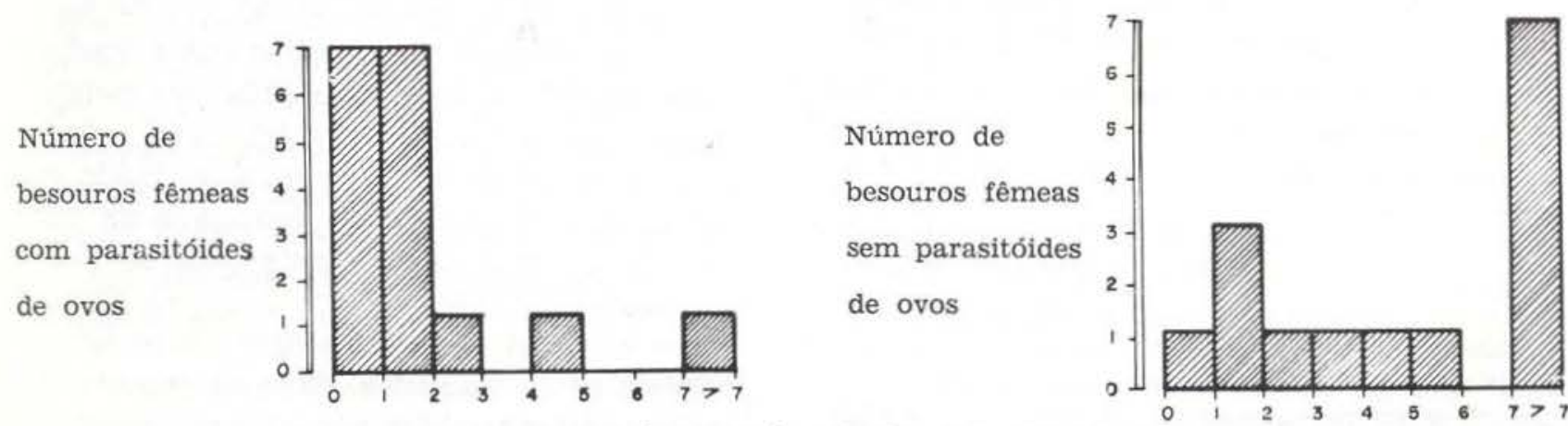

Dia em que os ovos foræm, pela primeira vez, depositados no laboratório.

FIGURA 1: Dia no qual as besouras Stolas, coletadas no campo, pela primeira vez depositaram ovos no laboratório. Besouras carregando parasitóides são comparadas com as besouras mais próximos que não estavam carregando parasitóides. (p 0,02, Kolmogorow - Simirnov, dois testes de amostra). 
nam foréticas sobre o primeiro besouro fêmea que elas encontram, e entăo permanecem com aquela besoura até que ela deposite seus ovos. Desde que a probabilidade de uma besoura atrair uma vespa é uma função do tempo disponivel para tais encontros ocorrerem, e desde que as gerações de besouros se superpõem. isto deveria explicar as observações de que as vespas parecem estar associadas com besouras mais velhas. Entretanto, há diversas razões pelas quais esta explicação é quase certamente inadequada: Primeiro, nem todas as besouras parecem semelhantes quanto ao acúmulo de vespas. A evidência é que diversos casos foram observados onde uma única besoura estava associada com duas ou mais vespas. Como foi afirmado anteriormente, a probabilidade de ocorrência de tais associações múltiplas como resultado de encontro ao acaso, dado ao grande número de besouras disponiveis, é extremamente pequena; Segundo, as vespas não formam associações contínuas com uma besoura. Uma vespa permanece com uma besoura por uma hora ou quase, e então voa, talvez em busca de néctar ou água. Por exempio, uma besoura, que foi observada du-

TABELA 7 - Produção de ovos por Stolas sp fê. mea coletada no campo. Besouras com parasitóides foréticos são comparadas com besouras mais próximos que carecem de parasitóides. (Testes: (1) T. de Student; (2) média; (3) T. de Student).

Besouros fêmeas Besouros fêmeas com parasitóides sem parasitóides

\begin{tabular}{|c|c|c|c|}
\hline \multicolumn{4}{|c|}{$\begin{array}{l}\text { Número médio de } \\
\text { ninhadas de ovos de }\end{array}$} \\
\hline Besouros & 15 & 15 & \\
\hline $\begin{array}{l}\text { Número médio de } \\
\text { ninhadas de ovos por } \\
\text { besoura poedeira }\end{array}$ & 4.57 & 3.44 & $P_{(z)}=0,058$ \\
\hline Besouros & 14 & 9 & \\
\hline $\begin{array}{l}\text { Número médio de } \\
\text { ovos por besoura }\end{array}$ & & & \\
\hline poedeira & 154,86 & 116,89 & N.S. (3) \\
\hline Besouros & 14 & 9 & \\
\hline
\end{tabular}

TABELA 8 - Número de fêmeas Stolas ainda vivas, depois de 36 dias de cativeiro.

Número de besouros

\begin{tabular}{lcr}
\hline & Vivo & Morto \\
\hline Besouros com parasitóides: & 1 & 14 \\
Besouros sem parasitóides: & 6 & 9 \\
\hline
\end{tabular}

rante um dia inteiro, não tinha vespas curante longos períodos e, em outras horas, tinha quatro vespas; Terceiro, no laboratório, tendio dispcniveis umidade e água açucarada, as vespas vivem somente uns poucos dias, enquanto que os besouros adultos podem viver mais do que 40 dias. Se as vespas no campo também têm uma vida adulta tão curta $€ m$ relação ao tempo de vida adulta de seu hospedeiro, então pouco valor adaptativo haveria para as vespas em formar uma associação com a primeira besoura encontrada por acaso. O intervalo entre a deposição de ovos para besouras de iaboratório é pelo menos 4 dias, e esse tempo é aproximadamente igual à duração de vespas adultas nas condições do laboratório.

$\mathrm{Se}$ as besouras hospedeiras fossem tão raras que o tempo de procura gasto peias vespas representasse uma fração significativa do tempo de vida de cada vespa, então a seieção deveria favorecer aquelas vespas que formassem associações com a primeira besoura encontrada. Neste estlido, vimos que Stolas sp ocorre em densidades tão aitas que a distância em linha reta entre fêmeas próximas raramente é mais que 1 metro e, além disso, o tempo de procura gasto pelas vespas é reduzido pela estrutura simples (pouca densidade de espécies de plantas) do ambiente onde as besouras são encontradas. Além disso, se o néctar é um alimento importante para essas vespas. então a periódica necessidade de procurar fintes de néctar também serviria como mediador contra a probabilidade de que as vespas formem associações por longo tempo com besouras encontradas ao acaso.

Como nãc conseguimos que essas vespas se associassem com besouras sob condições de laboratório, não pudemos fazer experimen- 
tos definitivos para mostrar claramente a natureza do processo de discriminação de hospedeiro. Entretanto, mesmo este breve estudo de campo traz evidências fortemente indicativas de um processo ativo de discriminação de hospedeiro.

\section{IMPLICAÇÕES PARA O CONTROLE BIOLÓGICO}

A fim de manter uma população contínua de inimigos naturais de insetos, que são pes. tes normalmente, é necessário fornecermos outras espécies de hospedeiros para o preciador ou parasitóide. Se tais fontes aiternativas de comida não forem disponíveis, as populaçoes de inimigos naturais podem tornar-se extintas localmente, quando os insetos-pestes tornarem-se, temporariamente, tão raros, que não suportem mais predadores ou parasitóides. Após a eliminação de seus inimigos naturais, a população de insetos-pestes podie, rapidamente, alcançar proporções destrutivas. O controle natural só voitará a ocorrer quandio os inimigos naturais recolonizarem a área. Portánto, espera-se que popuiações de insetos pestes escapem, periodicamente, à ação de seus inimigos naturais. Tais interações entre pestes versus inimigo são instáveis e caracterizadas por grandes flutuações na quantidade dos insetos-pestes.

Em sistemas de pequenas fazendas, nos trópicos, é muito importante desenvolver programas estáveis de controle biológico. O fazendeiro tropical não obterá recursos comerciais ou governamentais que the assegurem c fornecimento de inimigos naturais das pestes de plantações, no que difere funciamentalmente dos fazendeiros das zonas temperadas. Para proteção contra pestes, o fazendeiro tropical tem que depender de populações naturais de insetos benéficos, predadores e parasitóides.

A importância de estabelecer hospedeiros alternativos a fim de manter populações contínuas de insetos benéficos já foi sugerida por Van Emder (1964) e demonstrada experimentalmente por Doutt \& Nakate. (1973).

No presente estudo, ovos de Stolas $\mathrm{sp}$, que é uma espécie inóqua de besouro, poderiam desempenhar um papel importante como hos- pedieiro alternativo para Emersoneila neveipes uma parasitóide de ovos que também ataca Chelymorpha cassidea, que é um desioliador de batata-doce. Larvas de Stolas sp, no laboratório se alimentam e se desenvolvem com folhas frescas de batata-doce; entretanto, os aduitos recusam estas e essa espécie não foi cbservada em batata-doce durante este estudo. A situação requer estudos adicionais, poıs, bescuros Cassidinae, incluindo C. cassideae, podem ter um papel útil no controle da Convolvulus arvensis, uma erva daninha economicamente muito importante e largamente espalhada (Mohyuddin, 1969) .

\section{AGRADECIMENTOS}

Quero agradecer a Sra. Elza Suely de Carvalho Anderson pela tradução, e aos Srs. Anthony Anderson e Ricardo Braga pela leitura crítica do manuscrito. Sou também profundamente grato ao instituto Nacional de Pesquisas da Amazônia (INPA) pelo seu apoio neste estudo.

\section{Abstract}

The cassid beetle, Stolas sp., is an abundant leaf-eating herbivore, both as larva and adult, on the vine, Ipomoea asarifolia, a wild relative of the sweet potato. In the region around Manaus (Amazonas State, Brazil), an average of $86 \%$ of the egg clutches were completely parasitized by the eulophid, Emersonella neveipes. The wasp is phoretic on female beetles. In comparison with field beetles that lacked phoretic wasps, beetles in association with wasps (1) produced eggs sooner, (2) produced more egg clutches, and (3) had a shorter laboratory life expectancy. Host discrimination by the wasp is probably an active process rather than a passive association with randomly encountered beetles.

Emersonella neveipes also attacks Chelymorpha cassidea, a pest of sweet potato. Since Stolas sp. does not, as adults, feed on sweet potato foliage and does not occur in sweet potato fields, it could provide a useful innocuous alternate host for Emersonella neveipes. Since some species in the Convolvulaceae are important weeds in field crops, foliage-feeding cassid beetles may play a useful role in weed suppression. Any biological control program for the suppression of sweet potato pests may favor the spread of those noxious weeds that share the herbivore fauna of sweet potatoes. 


\section{BIBLIOGRAFIA CITADA}

BLACKWELDER, R.E.

1957 - Checklist of the Coleopterous insects of Mexico, Central America, the West Indies, and South America. United States National Museum Bulletin, 185 : 1-1492.

DOUTT, R.L. \& NAKATA, J.

1973 - The Rubus leafhopper and its egg parasitoid: An endemic biotic system useful in grape-pest management. Environmental Entomology, 2:381-386.
EMDEN, H.F, VAN

1965 - The role of uncultivated land in the biology of crop pests and beneficial insects. Scientific Horticulture, 17: 126-136.

FALCÃO, J.

1971 - Convolvulaceae do Amazonas. Acta Amazonica, 1(1) : 15-20.

MOHYUDDIN, A.I.

1969 - Insects from Calystegia spp. and Convolvulus spp. Commonwealth Institute of Biological Control, $11: 93-104$.

Silva, A.G.

1967 - Quatro catalogo dos insetos que vivem nas plantas do Brasil, seus parasitos e predatores. Rio de Janeiro, IBGE. 906 p. 